\title{
Adhärenz im Alter - Wie angehen?
}

Ungefähr 50\% der Allgemeinbevölkerung bedürfen einer Dauermedikation, bei Älteren (> 65) und Hochbetagten (> 85) sind es wahrscheinlich über $90 \%[5,10,11]$. Seit langem ist bekannt, dass die medikamentöse Adhärenz unzureichend ist: Ca. 50\% aller Menschen mit chronischen Erkrankungen nimmt eine Dauermedikation nicht wie verordnet ein; bei Älteren und Hochbetagten schwanken die Angaben zwischen $40-80 \%[5,10,11]$.

Über die letzten Jahrzehnte wurde ein differenziertes „bio-psycho-soziales“ Ursachenmodell der Non-Adhärenz entwickelt, das für Erwachsene allgemein gültig ist: Günstig auf die medikamentöse Adhärenz wirken sich ein einfaches Therapieschema und auf Patientenseite eine hohe Therapiemotivation, gutes Therapieverständnis, hohe Therapiezufriedenheit und gute soziale Unterstützung aus. Typischerweise stellen sich die vorgenannten Aspekte bei einer guten medizinischen Versorgung mit gleichermaßen motivierender wie verständlicher Arzt-PatientKommunikation sowie regelmäßigen Verlaufsterminen ein. Risikofaktoren für eine medikamentöse Non-Adhärenz sind ein kompliziertes Therapieschema (>3 unterschiedliche Tabletten, mehrere Einnahmezeitpunkte), geringes Therapieverständnis, Erleben von Nebenwirkungen (oder Angst davor), psychische Begleiterkrankungen (v.a. Depression), geringe soziale Unterstützung sowie niedriges Bildungsniveau $[2,11]$. Darüber hinaus wurden spezifisch für Ältere und Hochbetagte weitere Faktoren identifiziert, die das Risiko einer Non-Adhärenz teils vervielfachen können: Männliches Geschlecht, Multimorbidität, allgemeine Gebrechlichkeit, Einschränkungen des Sehens, Hörens und Tastens sowie, besonders wichtig, kognitive Einschränkungen bis zur Demenz [3,4, $6,13]$. Neben „intentionalen“ Faktoren für Non-Adhärenz (v.a. nicht motiviert für Therapie, Angst vor UAW) treten demnach bei Älteren oftmals „nicht-intentionale“ Faktoren (nicht verstanden, nicht behalten, nicht umsetzen können) in den Vordergrund.
Wirksame Interventionen zur allgemeinen Förderung der medikamentösen Adhärenz sind bekannt, die verfügbare Literatur für Ältere und Hochbetagte ist jedoch begrenzt. Allgemein werden eine Verringerung der Dosisanforderungen (Anzahl und Einnahmehäufigkeit) auf das niedrigste sinnvolle Niveau, regelmäßige Wiedervorstellungen und Patientenschulungen (kommunikative Förderung der Sinnhaftigkeit, Verstehbarkeit und Handhabbarkeit der Medikation) empfohlen [2,7,11]. Neuerdings ergibt sich auch eine gewisse Evidenz, dass neue Medien wie „Apps“ sinnvoll sein könnten [1]. Die suffiziente Behandlung einer etwaigen psychischen Komorbidität ist ein weiteres wesentliches Element [12].

Spezifisch für Ältere und Hochbetagte müssen die vorgenannten Ansätze jedoch bedarfsgerecht erweitert werden: Eine verständliche, motivierende Kommunikation, vorzugsweise ergänzt um kurze schriftliche Informationen, und ein möglichst einfaches Therapieschema (ggf. mit Kombinationspräparaten) bleiben die Basis. Ergänzend sollten körperliche und/ oder kognitive Kompetenzdefizite geklärt und berücksichtigt werden. Praktische Hilfen (z. B. Kalenderblister) haben sich bewährt und können bei Bedarf und Verfügbarkeit auch durch Angehörige befüllt werden [8, 9]. Zu dem Einsatz von „neuen Medien“ bei Älteren existieren nur einzelne Erfahrungsberichte; sie können bei Interesse zusätzlich erwogen werden. Bei ausgeprägtem Risiko sollte frühzeitig an eine häusliche Krankenpflege sowie ggf. Heimunterbringung gedacht werden.

\section{Interessenkonflikte}

Der Autor hat Referentenhonorare zu diesem Thema von Bayer Vital, Daiichi Sankyo und UCB erhalten. Ohne Bezug zu diesem Thema Referentenhonorare von Boehringer Ingelheim, Berlin-Chemie, PCO Tyrol Congress, MSD Sharp \& Dohme, der Akademie der DGK, DDG, WebMD Germany sowie vom Schattauer-Verlag, Elsevier und DÄV. Es bestehen keine sonstigen finanziellen Interessenkonflikte.
Autorinnen/Autoren

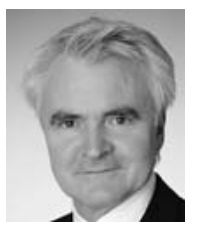

Christian Albus

Prof. Dr. med., FESC, Klinik und Poliklinik für Psychosomatik und Psychotherapie, Uniklinik Köln

\section{Korrespondenzadresse}

Prof. Dr. med. Christian Albus, FESC Klinik und Poliklinik für Psychosomatik und Psychotherapie

Uniklinik Köln

Kerpener Straße 62

50937 Köln

christian.albus@uk-koeln.de

Literatur

[1] Adler AJ, Martin N, Mariani J et al. Mobile phone textmessaging to improve medication adherence in secondary prevention of cardiovascular disease. Cochrane Database of Systematic Reviews 2017, Issue 4. Art. No.: CD011851.

[2] Albus C, Matthes J. Maßnahmen zur Förderung der Therapieadhärenz. MMW 2014 156: 54-55

[3] El-Saifi N, Moyle W, Jones C et al. Medication adherence in older adults with dementia: a systematic literature review. J Pharma Pract 2018; 31: 322-334

[4] Gellad WF, Grenard JL, Marcum ZA. A systematic review of barriers to medication adherence in the elderly: looking beyond cost and regimen complexity. Am J Geriatr Pharmacother 2011; 9: 11-23

[5] Hughes CM. Medication non-adherence in the elderly. How big is the problem? Drugs Aging 2004; 21: 793-811

[6] Jamerson BD, Fillenbaum GG, Sloane R et al. A new method for identifying characteristics of needing help to take medications in an older representative community-resident population: The Duke Medication Assist Scale. J Am Geritr Soc 2016; 64: 1195-1202

[7] Kripalani S, Yao X, Haynes RB. Interventions to enhance medication adherence in chronic medical conditions. A systematic review. Arch Int Med 2007; 167: 540-550

[8] Kröger E, Tatar O, Vedel I et al. Improving medication adherence among communitydwelling seniors with cognitive impairment: a systematic review of interventions. Int J Clin Pharm 2017; 39: 641-656 
[9] Marcum ZA, Hanlon JT, Murray MD. Improving medication adherence and health outcomes in older adults: An evidence-based review of randomized controlled trials. Drugs Aging 2017; 34: 191-201

[10] Morin L, Vetrano DL, Rizzuto D et al. Choosing wisely? Measuring the burden of medica- tions in older adults near end of life: nationwide, longitudinal cohort study. Am J Med 2017; 130: 927-936

[11] Osterberg L, Blaschke T. Adherence to medication. N Engl J Med 2005; 353: 487-497

[12] Petrak F, Meier JJ, Albus C et al. Motivation und Diabetes - Zeit für einen Paradigmen- wechsel? Ein Positionspapier. Diabetologie und Stoffwechsel 2019; 14: 193-203

[13] Yap AF, Thirumoorthy T, Kwan YH. Systematic review of the barriers affecting medication adherence in older adults. Geriatr Gerontol Int 2016; 16: 1039-1101 